\title{
Agrobiodiversidade de sistemas agroflorestais com cafeeiro na Amazônia ocidental
}

Os agroecossistemas de base agroecológica contribuem com a mitigação dos problemas ambientais, ao mesmo tempo que produzem bens e serviços ecossistêmicos. Objetivou-se investigar a composição florística, riqueza e diversidade de plantas em sistemas agroflorestais (SAF) com cafeeiro canéfora (Coffea sp.), como componente principal, estabelecidos no município de Cacoal, Rondônia. Foram inventariados todos os indivíduos com diâmetro a altura do peito maior ou igual a cinco $\mathrm{cm}$, medidos a $130 \mathrm{~cm}$ do solo, em sete SAF. Após a identificação das espécies foram calculados os parâmetros de frequência, densidade, dominância, valor de importância, valor de cobertura, similaridade de Jaccard, diversidade de Shannon-Wierner, equabilidade de Pielou e dominância de Simpson. Para a estrutura vertical, os SAF foram estratificados pelo método de Longhi. Foram inventariados 835 indivíduos, distribuídos em 71 espécies, 5 morfoespécies, 54 gêneros e 27 famílias botânicas. As famílias com maior riqueza de espécies foram: Fabaceae, Annonaceae, Myrtaceae, Anacardiaceae e Bignoniaceae. Os SAF apresentaram em sua composição florística gradiente de espécies, com $49 \%$ de nativas, $23 \%$ exóticas e $28 \%$ não foram confirmadas. Os SAF são pouco semelhantes entre si, com nenhum espécie comum a todos. O índice de diversidade de Shannon-Wierner, variou de 0,54 a 2,79; equabilidade de Pielou 0,42 a 0,87 e dominância de Simpson 0,27 a 0,92. As espécies mais frequentes foram embaúba (Cecropia engleriana Snethl.) e bandarra (Schizolobium parahyba var. amazonicum (Huber ex Ducke Barneby), presentes em $86 \%$ dos SAF. 0 valor de importância das espécies que compõem os SAF, mostrou um estoque de indivíduos com potencial madeireiro. Os SAF apresentaram-se multiestratificados, com presença de três estratos para todos os agroecossistemas. Em 43\% dos SAF os indivíduos melhor representados estão no estrato dois (E2). Os coeficientes encontrados indicam os SAF com cafeeiro com potencial para a conservação da natureza e da agrobiodiversidade.

Palavras-chave: Agroecossistemas Tropicais; Agricultura Sustentável; Café.

\section{Agrobiodiversity of agroforestry systems with coffee in western Amazonia}

\begin{abstract}
Agroecosystems based on agroecology contribute to the mitigation of environmental problems, while producing ecosystem goods and services. The objective of this study was to investigate the floristic composition, richness and diversity of plants in agroforestry systems (SAF) with canephora coffee tree (Coffea sp.), as main component, established in the municipality of Cacoal, Rondônia. All individuals with a breast height diameter greater than or equal to five $\mathrm{cm}$, measured at $130 \mathrm{~cm}$ from the ground, were inventoried in seven $S A F$. After identification of the species were calculated the parameters of frequency, density, dominance, importance value, coberture value, Jaccard similarity, Shannon-Wierner diversity, Pielou equability and Simpson dominance. For the vertical structure, the SAF were stratified by the Longhi method. A total of 835 individuals, distributed in 71 species, 5 morphospecies, 54 genera and 27 botanical families, were inventoried. The families with the highest species richness were: Fabaceae, Annonaceae, Myrtaceae, Anacardiaceae e Bignoniaceae. The SAF presented in their floristic composition a species gradient, with $49 \%$ of native, $23 \%$ exotic and $28 \%$ were not confirmed. SAF are not similar, with no common species at all. The index diversity of Shannon-Wierner, ranged form 0,54 to 2,79; Pielou equability from 0,42 to 0,87 and Simpson dominance 0,27 to 0,92. The species most frequent were embaúba (Cecropia engleriana Snethl.) e bandarra (Schizolobium parahyba var. amazonicum (Huber ex Ducke Barneby), present in $86 \%$ of the SAF. The importance value of the species that make up the SAF, showed a stock of individuals with potential timber. The SAF were multi-stratified, with presence of three strata for all agroecosystem. In $43 \%$ of the SAF the best represented individuals are in stratum two (E2). The coefficients found indicate the SAF with coffee tree with potential for nature conservation and agrobiodiversity.
\end{abstract}

Keywords: Tropical Agroecosystems; Sustainable Agriculture; Coffee.

Topic: Conservação da Biodiversidade

Reviewed anonymously in the process of blind peer.

Maria Cristiana de Freitas da Costa

Instituto Federal de Educação, Ciência e Tecnologia de Rondônia, Brasil http://lattes.cnpq.br/5077293669107785

http://orcid.org/0000-0001-7914-4987

crisfreitasczs@gmail.com

Giordano Bruno da Silva Oliveira (iD)

Universidade Federal do Acre, Brasil

http://lattes.cnpq.br/6921152217766876

http://orcid.org/0000-0003-2642-711X

giordano.silva@gmail.com

Anna Frida Hatsue Modro (iD

Universidade Federal de Rondônia, Brasil

http://lattes.cnpq.br/9474501340526969

http://orcid.org/0000-0002-6201-960X

anna.frida@unir.br

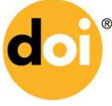

DOI: 10.6008/CBPC2179-6858.2018.002.0008
Received: $14 / 12 / 2017$

Approved: 24/01/2018

Fernando Ferreira de Morais

Universidade Federal do Recôncavo da Bahia, Brasil

http://lattes.cnpq.br/8626627645181352

moraisff@hotmail.com

André de Paulo Evaristo (iD

Universidade Federal de Rondônia, Brasil

http://lattes.cnpq.br/0612300232180051

http://orcid.org/0000-0003-1874-5855

andrepauloevaristo@hotmail.com

Emanuel Fernando Maia de Souza it

Universidade Federal de Rondônia, Brasil

http://lattes.cnpq.br/4742387914768064

http://orcid.org/0000-0002-5493-2183

emanuel@unir.br

Referencing this:

COSTA, M. C. F.; OLIVEIRA, G. B. S.; MODRO, A. F. H.; MORAIS, F. F.; EVARISTO, A. P.; SOUZA, E. F. M.. Agrobiodiversidade de sistemas agroflorestais com cafeeiro na Amazônia ocidental. Revista Ibero Americana de Ciências Ambientais, v.9, n.2, p.84-93, 2018. DOI: http://doi.org/10.6008/CBPC2179-6858.2018.002.0008 


\section{INTRODUÇÃO}

Sistemas agroflorestais (SAF) são formas de uso e manejo dos recursos naturais, onde árvores, arbustos e palmeiras são associadas com culturas agrícolas ou animais na propriedade, simultaneamente ou em uma sequência temporal (MONTAGNINI et al., 1992). As paisagens agroflorestais tropicais são configurações extremamente dinâmicas, podendo apresentar intensidade, produtividade e capacidade diferentes (HARRINGTON et al., 2012). Nestes sistemas, cada árvore apresenta crescimento conforme as características endógenas da espécie e de acordo com as condições ofertadas pelo ecossistema, em período e velocidades diferentes, construindo sistemas chamados de 'matas' construídas (LEÃO et al., 2017). Em ambientes assim, estudos florísticos e fitossociológicos permitem vislumbrar quali-quantitativamente as espécies presentes e suas peculiaridades, podendo ser ferramenta para manejo, conhecimento de potencial madeireiro e não madeireiro e da própria caracterização do sistema. Do ponto de vista da conservação e prestação de serviços ambientais, são associados aos SAF: redução da pressão de exploração sobre áreas protegidas, servindo de refúgio da biodiversidade, contribuindo para a manutenção da heterogeneidade do ambiente, conservação dos solos, qualidade do ar e sequestro de carbono (MURNIATI et al., 2001; BHAGWAT et al., 2008).

De modo intrínseco aos SAF, essas pesquisas podem aprovisionar subsídios a respeito das relações entre indivíduos arbóreos e os fatores ambientais, contribuindo para o estado da arte e indicando espécies mais adequadas para cada agroecossistema e região (LEÃO et al., 2017). No mais, análises estruturais e florísticas possibilitam a geração de um sistema de classificação que diferenciam os estágios vegetativos de sistemas silviagrícolas (BOLFE et al., 2011). Assim, objetivou-se investigar a composição florística, riqueza e diversidade de plantas em sistemas agroflorestais (SAF) com cafeeiro canéfora (Coffea sp.), como componente principal, estabelecidos no município de Cacoal, Rondônia.

\section{METODOLOGIA}

Foram amostrados sete sistemas agroflorestais com cafeeiro (Coffea sp.), localizados na linha 10, zona rural do município de Cacoal, no estado de Rondônia. De acordo com a classificação climática de Köppen-Geiger, o clima da região é do tipo Am (tropical de monção), com temperatura média anual entre 24 e $26{ }^{\circ} \mathrm{C}$ e precipitação média anual entre 2000 a $3000 \mathrm{~mm}$ (ALVARES et al., 2014). A tipologia florestal predominante da região, caracteriza-se por Floresta Ombrófila Densa Submontana (OLIVEIRA, 2005). Os solos predominantes são, Latossolo Litólico Distrófico, Argissolo Vermelhos Eutrófico e Neossolo Eutrófico (CASAGRANDE, 2009).

Os SAF classificados pelas letras A, B, C, D e E localizam-se no sítio Três Irmãos (W 61을 20' 13.06" e S 11 21' 50.36"). Esses sistemas foram implantados no ano de 2004. O componente arbóreo não possui um espaçamento padrão e está distribuído em diferentes intensidades nos sistemas. Além das espécies escolhidas e introduzidas intencionalmente, esses sistemas agroflorestais são enriquecidos por espécies oriundas da regeneração natural. O manejo do cafeeiro é frequente e do componente arbóreo é ocasional. 
Na Linha 10, Sítio Boa Vista (W 610 19' 52.16" e S 11 17' 55.08'), estão localizados os SAF F e G. O primeiro foi implantado, no ano de 1978, em uma área de pastagem já estabelecida e o segundo substituiu uma lavoura de mamão, no ano de 2011. Ambos os sistemas não recebem tratos culturais frequentemente, o que favorece a regeneração natural, principalmente no SAF G que recebe aporte de sementes do SAF F e fragmentos florestais próximos.

Para conhecimento da composição florística, riqueza e diversidade de plantas dos sistemas foram inventariados todos os indivíduos com diâmetro a altura do peito maior ou igual a cinco cm, medidos a 130 cm do solo com auxílio de fita métrica, e a altura foi estimada, seguindo o proposto por Soares et al. (2012). As áreas de SAF amostradas não ultrapassaram o limite de um hectare cada uma.

As características dendrológicas dos componentes foram anotadas em ficha de campo. A identificação dos indivíduos foi realizada in loco, com o auxílio dos agricultores, manuais de identificação e coleta de material botânico. As exsicatas foram incorporadas ao acervo do Laboratório de Horticultura Tropical e Apicultura da Universidade Federal de Rondônia - LAHorTA. O sistema de classificação adotado foi o Angiosperm Phylogeny Group. Posteriormente, para se confirmar a grafia correta dos nomes científicos e dos autores de cada espécie, foram acessados a base de dados do site Tropicos.

Na análise da estrutura horizontal dos SAF foram calculados os parâmetros relativos e absolutos de frequência, densidade e dominância, valor de importância das espécies e valor de cobertura (MUELLERDOMBOIS et al., 1974; MARTINS, 1991; LAMPRECHT, 1964). Para análise de similaridade, diversidade, dominância e equabilidade dos sistemas agroflorestais, adotou-se o índice de similaridade de Jaccard (SJ), índice de diversidade de Shannon-Wierner $\left(\mathrm{H}^{\prime}\right)$, equabilidade de Pielou (J) e dominância de Simpson (C) (BROWER et al., 1984; PERKINS, 1982; HUTCHESON, 1970).

$\mathrm{Na}$ avaliação da estrutura vertical dos sistemas agroflorestais, empregou-se o método de Longhi (1980), que divide em três partes iguais a frequência relativa das alturas encontradas nos SAF. Adotou-se a fórmula de Sturges (1926), para definir o intervalo de classe: IC = A/nc, em que A é a amplitude de classe e $\mathrm{nc}=1+3,322\left(\log _{10} \mathrm{n}\right)$ em que $\mathrm{n}$ é o número de indivíduos. Ao final, foi gerada uma tabela com os estratos presentes nos sistemas agroflorestais.

\section{RESULTADOS}

Foram inventariados 835 indivíduos nos sete SAF, distribuídos em 71 espécies, 5 morfoespécies, 54 gêneros e 27 famílias botânicas. Os sistemas agroflorestais com cafeeiro apresentaram em sua composição florística gradientes de espécies variados, onde $49 \%$ são nativas, $23 \%$ são exóticas e $28 \%$ não foram confirmadas (Tabela 1).

Tabela 1: Composição florística dos sete sistemas agroflorestais com cafeeiro, localizados no município de Cacoal, Rondônia.

\begin{tabular}{llc}
\hline Família/Espécie & Nome Comum & Origem \\
\hline Anacardiaceae & & Ocorrência (SAF) \\
Anacardium occidentale L. & Caju & N \\
Astronium lecointei Ducke & Aroeira & N \\
Mangifera indica L. & Mangueira & E
\end{tabular}




\begin{tabular}{|c|c|c|c|}
\hline Spondias mombin L. & Cajá & $\mathrm{N}$ & G \\
\hline Spondias purpurea L. & Seriguela & $\mathrm{E}$ & G \\
\hline \multicolumn{4}{|l|}{ Annonaceae } \\
\hline Annona muricata $\mathrm{L}$. & Graviola & $\mathrm{E}$ & $C, E$ \\
\hline Annona sp. & - & - & $\mathrm{F}$ \\
\hline Duguetia sp. & Cacau de índio & - & $\mathrm{E}$ \\
\hline Guatteria sp. 1 & Pindaíba preta & - & A \\
\hline Guatteria sp. 2 & - & - & $\mathrm{F}$ \\
\hline Guatteria sp. 3 & - & - & $\mathrm{F}$ \\
\hline \multicolumn{4}{|l|}{ Apocynaceae } \\
\hline Aspidosperma polyneuron Müll.Arg. & Peroba rosa & $\mathrm{N}$ & $\mathrm{E}$ \\
\hline Aspidosperma sandwithianum Markgr. & Peroba rosa & $\mathrm{N}$ & $A$ \\
\hline Tabernamontana sp. & - & - & $\mathrm{F}$ \\
\hline \multicolumn{4}{|l|}{ Arecaceae } \\
\hline Bactris gasipais Kunth & Pupunheira & $\mathrm{N}$ & $\mathrm{C}$ \\
\hline Cocos nucifera L. & Coqueiro & $\mathrm{E}$ & $C, E$ \\
\hline \multicolumn{4}{|l|}{ Bignoniaceae } \\
\hline Handroanthus Serratifolius (Vahl.) S.Grose & Ipê amarelo & $\mathrm{N}$ & G \\
\hline Handroanthus sp. & Ipê & - & $\mathrm{F}, \mathrm{G}$ \\
\hline Jacaranda copaia (Aubl.) D.Don & Caroba & $\mathrm{N}$ & A \\
\hline Tabebuia sp. 1 & Ipê & - & $C, D, F, G$ \\
\hline Tabebuia sp. 2 & Ipê preto & - & $\mathrm{G}$ \\
\hline \multicolumn{4}{|l|}{ Bixaceae } \\
\hline Bixa orellana $\mathrm{L}$. & Urucum & $\mathrm{N}$ & $\mathrm{F}$ \\
\hline \multicolumn{4}{|l|}{ Boraginaceae } \\
\hline Cordia sellowiana Cham. & - & $\mathrm{N}$ & A \\
\hline \multicolumn{4}{|l|}{ Caryocaraceae } \\
\hline Caryocar villosum (Aubl.) Pers. & Pequi preto & $\mathrm{N}$ & A \\
\hline \multicolumn{4}{|l|}{ Chrysobalanaceae } \\
\hline Licania tomentosa Benth. & Oiti & $\mathrm{N}$ & $\mathrm{E}$ \\
\hline
\end{tabular}

Licania tomentosa Benth.

\begin{tabular}{|c|c|c|}
\hline Família/Espécie & Nome Comum & Origem \\
\hline \multicolumn{3}{|l|}{ Euphorbiaceae } \\
\hline Hevea brasiliensis (Willd. ex A.Juss.) Müll.Arg. & Seringueira & $\mathrm{N}$ \\
\hline Sapium glandulosum (L.) Morong & - & $\mathrm{N}$ \\
\hline
\end{tabular}

\section{Fabaceae}

Bauhinia variegata $\mathrm{L}$.

Chamaecrista adiantifolia (Spruce ex Benth.) H.S.Irwin \& Barneby

Tamarindus indica $\mathrm{L}$.

Pata de vaca

Baginha

Tamarino

Imburana

Taralea sp.

Enterolobium schomburgkii (Benth.) Benth.

Inga edulis Mart.

Parkia sp. 1

Parkia sp. 2

Samanea tubulosa (Benth.) Barneby \& J.W.Grimes

Schizolobium parahyba var. amazonicum (Huber ex Ducke) Barneby

\section{Hypericaceae}

Vismia guianensis (Aubl.) Choisy

Vismia sp.

Lamiaceae

Tectona grandis L. f.

Lauraceae

Aniba parviflora (Meisn.) Mez

Cinnamomum sp.

Mezilaurus itauba (Meisn.) Taub. ex Mez

Lecythidaceae

Couratari stellata A.C.Sm.

\section{Malvaceae}

Ceiba pentandra (L.) Gaertn.

Ceiba speciosa (A.St.-Hil.) Ravenna

Theobroma cacao L.

Theobroma grandiflorum (Willd. ex Spreng.) K.Schum.

\section{Meliaceae}

Cedrela fissilis Vell.

Swietenia macrophylla King
Jatobazinho

Baginha vermelha $\mathrm{N} \quad \mathrm{F}$

Ingá N $\quad \mathrm{C}, \mathrm{E}$

Faveira de paca $\quad-\quad$ F

Pinho - $\quad$ G

Mel N E, F

Bandarra

$\mathrm{N}$

$A, B, D, F, G$

$\begin{array}{lll}- & N & A\end{array}$

Teca

E

A, C, D, E

Louro N E

Itaúba

$\mathrm{N}$

G

Embirema

N A

Samaúma

$\mathrm{N}$

A, C, F, G

Paineira

N

Cacau

E

$E, F, G$

Cupuaçu

N $\quad$ E

$\begin{array}{lll}\text { Cedro } & \text { N } & \text { E, F, G } \\ \text { Mogno } & \text { N } & \text { A, C, D, G }\end{array}$




\begin{tabular}{|c|c|c|c|c|}
\hline \multicolumn{5}{|l|}{ Moraceae } \\
\hline Artocarpus heterophyllus Lam. & \multicolumn{2}{|r|}{ Jaca } & $\mathrm{E}$ & $A, C, E, G$ \\
\hline Ficus sp. 1 & & Apuí & - & $\mathrm{F}$ \\
\hline Ficus sp. 2 & & Figueira & - & $\mathrm{F}$ \\
\hline Maclura tinctonia (L.) D.Don ex Steud. & & Moreira & $\mathrm{N}$ & $E, G$ \\
\hline \multicolumn{5}{|l|}{ Myrtaceae } \\
\hline Eugenia uniflora $\mathrm{L}$. & & Pitanga & $\mathrm{N}$ & $E$ \\
\hline Plinia cauliflora (DC.) Kausel & & Jabuticabeira & $\mathrm{N}$ & $\mathrm{C}$ \\
\hline Plinia jaboticaba (Vell.) Kausel & & Jabuticabeira & $\mathrm{N}$ & $\mathrm{E}$ \\
\hline Psidium guajava $\mathrm{L}$. & & Goiabeira & $E$ & $C, E$ \\
\hline Syzygium cumini (L.) Skeels & & Jamelão & $E$ & $E$ \\
\hline Syzygium jambos (L.) Alston & & Jambo & $\mathrm{E}$ & $\mathrm{E}$ \\
\hline \multicolumn{5}{|l|}{ Rhamnaceae } \\
\hline Hovenia dulcis Thunb. & & Uva japonesa & $\mathrm{E}$ & $\mathrm{E}$ \\
\hline Família/Espécie & Nome Comum & Origem & \multicolumn{2}{|c|}{ Ocorrência (SAF) } \\
\hline \multicolumn{5}{|l|}{ Rubiaceae } \\
\hline Genipa americana L. & Jenipapo & $\mathrm{N}$ & \multicolumn{2}{|c|}{$F, G$} \\
\hline Rustia sp. & Sobrasil & - & G & \\
\hline \multicolumn{5}{|l|}{ Rutaceae } \\
\hline \multirow[t]{2}{*}{ Citrus reticulata Blanco } & Pokam & $E$ & \multirow{2}{*}{\multicolumn{2}{|c|}{$E, F$}} \\
\hline & Tangerina & & & \\
\hline Citrus sinensis (L.) Osbesck & Laranjeira & $E$ & $\mathrm{E}$ & \\
\hline Citrus x limonia (L.) Osbeck & Limão tangerina & $E$ & $\mathrm{D}$ & \\
\hline \multirow[t]{2}{*}{ Zanthoxylum rhoifolium Lam. } & Mamica de cadela & $\mathrm{N}$ & \multicolumn{2}{|c|}{$A, F, G$} \\
\hline & Mamica de porca & & & \\
\hline \multicolumn{5}{|l|}{ Sapotaceae } \\
\hline Pouteria sp. 1 & Abiu do mato & - & \multicolumn{2}{|l|}{$E$} \\
\hline Pouteria sp. 2 & - & - & $\mathrm{A}$ & \\
\hline \multicolumn{5}{|l|}{ Urticaceae } \\
\hline Cecropia engleriana Snethl. & Embaúba & $\mathrm{N}$ & \multicolumn{2}{|c|}{$A, B, C, D, E, G$} \\
\hline
\end{tabular}

Onde: $\mathrm{N}$ = nativa e $\mathrm{E}=$ exótica. $\mathrm{A}, \mathrm{B}, \mathrm{C}, \mathrm{D}, \mathrm{E}, \mathrm{F}$ e $\mathrm{G}=$ sistemas agroflorestais amostrados.

As famílias com maior riqueza de espécies foram: Fabaceae, Annonaceae, Myrtaceae, Anacardiaceae e Bignoniaceae (Figura 1). A média de espécies por SAF foi de 10,85. A maior riqueza foi encontrada no SAF E a menor no SAF B, com 29 e 3 espécies, respectivamente. A família Arecaceae, contribuiu com $4 \%$ da riqueza de espécies presente nos agroecossistemas com cafeeiro. No total, 30\% das famílias foram representados por apenas uma espécie.

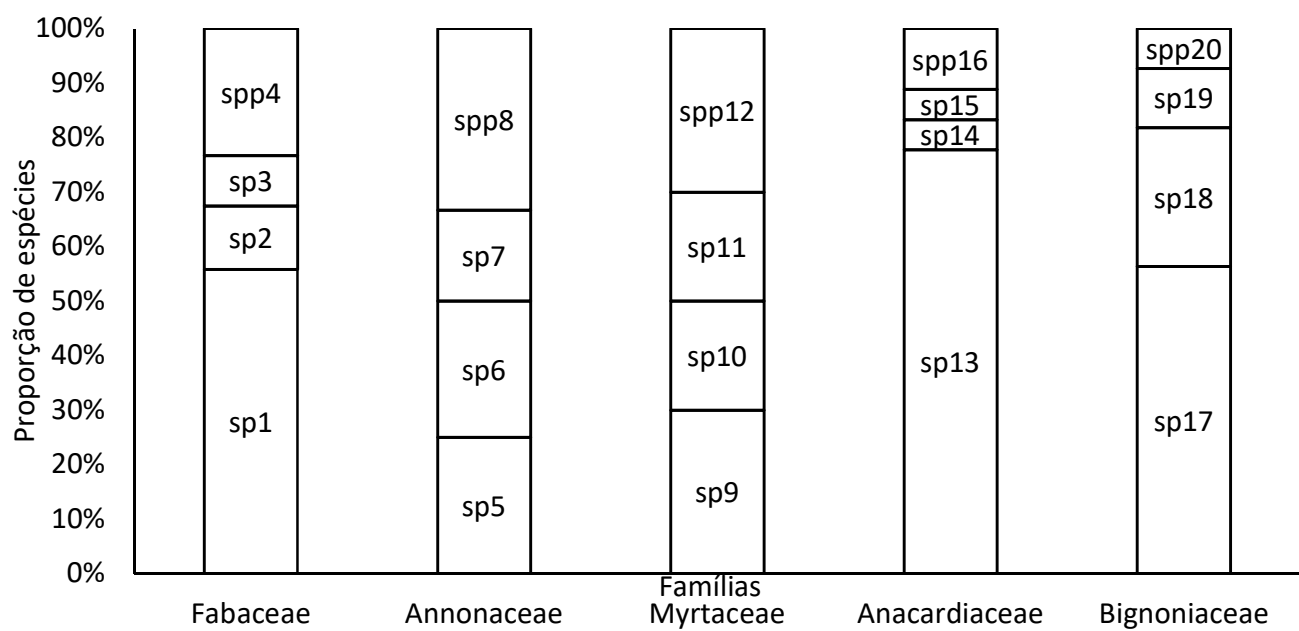

Figura 1: Famílias botânicas com maior percentual de riqueza de espécies para os sete sistemas agroflorestais com cafeeiro do município de Cacoal, Rondônia. Legenda: sp1 = Schizolobium parahyba var. amazonicum (Huber ex Ducke) Barneby; sp2 = Amburana sp.; sp3 = Samanea tubulosa (Benth.) Barneby \& J. W. Grimes; spp4 = Outras 12 espécies.; sp5 = Annona muricata L.; sp6 = Annona sp.; sp7 = Guatteria sp.; spp8 = Outras três espécies; sp9 = Plinia jaboticaba (Vell.) Kausel; sp10 = Psidium

guajava L.; sp11 = Syzygium cumini (L.) Skeels; spp12 = Outras três espécies; sp13 = Mangifera indica L.; sp14 = Anacardium occidentale L.; sp15 = Astronium lecointei Ducke; spp16 = Outras duas espécies; sp17 = Tabebuia sp.; sp18 = Handroanthus sp.; sp19 = Handroanthus Serratifolius (Vahl.) S. Grose; spp20 = Outras duas espécies. 
Nas famílias com os maiores percentuais de riqueza de espécies, destacaram-se pela densidade, em ordem decrescente, as espécies: bandarra (Schizolobium parahyba var. amazonicum), graviola (Annona muricata), jabuticabeira (Plinia jaboticaba), mangueira (Mangifera indica) e ipê (Tabebuia sp.). Em 43\% dos SAF, o índice de diversidade de Shannon-Wiener ficou acima de $\mathrm{H}^{\prime}=2,00$, com dominância de Simpson acima de $C=0,80$ e de equabilidade de Pielou superior a 0,70. O SAF G apresentou os maiores valores para todos os índices (Tabela 2).

Tabela 2: Valores de diversidade de Shannon-Wierner ( $\left.\mathrm{H}^{\prime}\right)$, equabilidade de Pielou (J) e dominância de Simpson (C) para os sete sistemas agroflorestais do município Cacoal, Rondônia.

\begin{tabular}{llll}
\hline Índices & $\boldsymbol{H}^{\prime}$ & $\boldsymbol{J}$ & $\boldsymbol{C}$ \\
\hline SAF & 1,25 & 0,42 & 0,54 \\
B & 0,54 & 0,49 & 0,27 \\
C & 1,63 & 0,62 & 0,74 \\
D & 1,31 & 0,63 & 0,64 \\
E & 2,49 & 0,74 & 0,84 \\
F & 2,65 & 0,85 & 0,90 \\
G & 2,79 & 0,87 & 0,92 \\
\hline
\end{tabular}

O índice de similaridade mostrou baixa semelhança entre os agroecossistemas. Sendo mais similares os SAF C e D, C e E, ambos com os mesmos valores (Tabela 3).

Tabela 3: Matriz do índice de similaridade de Jaccard (SJ) dos sete sistemas agroflorestais com cafeeiro do município de Cacoal, Rondônia.

\begin{tabular}{|c|c|c|c|c|c|c|c|}
\hline SAF & A & B & C & D & $\mathbf{E}$ & $\mathbf{F}$ & G \\
\hline A & 1 & 0,10 & 0,22 & 0,22 & 0,09 & 0,08 & 0,15 \\
\hline B & - & 1 & 0,07 & 0,22 & 0,07 & 0,04 & 0,12 \\
\hline C & - & - & 1 & 0,24 & 0,24 & 0,06 & 0,15 \\
\hline D & - & - & - & 1 & 0,09 & 0,07 & 0,14 \\
\hline$E$ & - & - & - & - & 1 & 0,08 & 0,13 \\
\hline $\mathbf{F}$ & - & - & - & - & - & 1 & 0,2 \\
\hline G & - & - & - & - & - & - & 1 \\
\hline
\end{tabular}

Destacaram-se por densidade relativa (DR superior a 3) as espécies Tectona grandis, Swietenia macrophylla, Mezilaurus itauba, Schizolobium parahyba var. amazonicum, Tabebuia sp. e Cecropia engleriana Snethl (Tabela 4).

Tabela 4: Listagem de espécies e os respectivos SAF de ocorrência (Ui), frequência absoluta (FA), frequência relativa (FR), densidade absoluta (DA), densidade relativa (DR), dominância absoluta (DoA), dominância relativa (DoR), valor de importância (VI) e valor de cobertura para os sete sistemas agroflorestais do município de Cacoal, Rondônia.

\begin{tabular}{|c|c|c|c|c|c|c|c|c|c|}
\hline Espécies & Ui & FA & FR & DA & DR & DoA & DoR & VI & VC \\
\hline Amburana sp. & 1 & 0,14 & 0,82 & 1,43 & 1,20 & 0,55 & 6,94 & 2,99 & 4,07 \\
\hline Anacardium occidentale L. & 1 & 0,14 & 0,82 & 0,14 & 0,12 & 0,00 & 0,02 & 0,32 & 0,07 \\
\hline Aniba parviflora (Meisn.) Mez. & 1 & 0,14 & 0,82 & 0,29 & 0,24 & 0,02 & 0,27 & 0,44 & 0,25 \\
\hline Annona muricata $\mathrm{L}$. & 2 & 0,29 & 1,64 & 0,43 & 0,36 & 0,03 & 0,42 & 0,80 & 0,39 \\
\hline Annona sp. & 1 & 0,14 & 0,82 & 0,43 & 0,36 & 0,02 & 0,23 & 0,47 & 0,29 \\
\hline Artocarpus heterophyllus Lam. & 4 & 0,57 & 3,28 & 0,71 & 0,60 & 0,10 & 1,29 & 1,72 & 0,94 \\
\hline Aspidosperma polyneuron Müll. Arg. & 1 & 0,14 & 0,82 & 0,14 & 0,12 & 0,02 & 0,21 & 0,38 & 0,16 \\
\hline Aspidosperma sandwithianum Markgr. & 1 & 0,14 & 0,82 & 0,14 & 0,12 & 0,00 & 0,04 & 0,33 & 0,08 \\
\hline Astronium lecointei Ducke & 1 & 0,14 & 0,82 & 0,14 & 0,12 & 0,02 & 0,30 & 0,41 & 0,21 \\
\hline Bactris gasipais Kunth & 1 & 0,14 & 0,82 & 0,43 & 0,36 & 0,02 & 0,30 & 0,49 & 0,33 \\
\hline Bauhinia variegata $\mathrm{L}$. & 2 & 0,29 & 1,64 & 0,43 & 0,36 & 0,00 & 0,05 & 0,68 & 0,20 \\
\hline Bixa orellana $\mathrm{L}$. & 1 & 0,14 & 0,82 & 0,14 & 0,12 & 0,00 & 0,03 & 0,32 & 0,07 \\
\hline Caryocar villosum (Aubl.) Pers. & 1 & 0,14 & 0,82 & 0,14 & 0,12 & 0,00 & 0,04 & 0,33 & 0,08 \\
\hline Cecropia engleriana Snethl. & 6 & 0,86 & 4,92 & 3,71 & 3,11 & 0,10 & 1,27 & 3,10 & 2,19 \\
\hline Cedrela fissilis Vell. & 3 & 0,43 & 2,46 & 1,57 & 1,32 & 0,07 & 0,87 & 1,55 & 1,09 \\
\hline
\end{tabular}


Ceiba pentandra (L.) Gaertn.

Ceiba speciosa (A. St.-Hil.) Ravenna

Chamaecrista adiantifolia (Spruce ex Benth.) H. S. Irwin \& Barneby Cinnamomum sp.

Citrus sinensis (L.) Osbesck

Citrus x limonia (L.) Osbeck

Cocos nucifera $\mathrm{L}$.

Cordia sellowiana Cham.

Couratari stellata A. C. Sm.

Duguetia sp.

Enterolobium schomburgkii (Benth.) Benth.

Eugenia uniflora $\mathrm{L}$.

Ficus sp. 1

Ficus sp. 2

Genipa americana L.

Guatteria sp. 1

Guatteria sp. 2

Guatteria sp. 3

Handroanthus Serratifolius (Vahl.) S. Grose

Handroanthus sp.

Hevea brasiliensis (Willd. ex A. Juss.) Müll. Arg.

Hovenia dulcis Thunb.

Inga edulis Mart.

Jacaranda copaia (Aubl.) D. Don

Licania tomentosa Benth.

Maclura tinctonia (L.) D. Don ex Steud.

Mangifera indica $\mathrm{L}$.

Mezilaurus itauba (Meisn.) Taub. ex Mez.

Parkia sp. 1

Parkia sp. 2

Plinia cauliflora (DC.) Kausel

Plinia jaboticaba (Vell.) Kausel

Pouteria sp. 1

Pouteria sp. 2

Psidium guajava L.

Rustia sp.

Samanea tubulosa (Benth.) Barneby \& J. W. Grimes

Sapium glandulosum (L.) Morong

Schizolobium parahyba var. amazonicum (Huber ex Ducke) Barneby 6

Spondias mombin L.

Spondias purpurea $\mathrm{L}$.

Swietenia macrophylla King.

Syzygium cumini (L.) Skeels

Tabebuia sp. 1

Tabebuia sp. 2

Tamarindus indica $\mathrm{L}$.

Taralea sp.

Tectona grandis L. f.

Theobroma cacao L.

Theobroma grandiflorum (Willd. ex Spreng.) K. Schum.

Vismia guianensis (Aubl.) Choisy

Vismia sp.

Zanthoxylum rhoifolium Lam.

Morfotipo 1

Morfotipo 2

Morfotipo 3

Morfotipo 4

Morfotipo 5
Citrus reticulata Blanco

Syzygium jambos (L.) Alston

Tabernamontana sp.

\begin{tabular}{|c|c|c|c|c|c|c|c|}
\hline & & & & & & & \\
\hline & & & & & & & 69 \\
\hline & 82 & 71 & 50 & 09 & & & \\
\hline 14 & 82 & 14 & 12 & 00 & 03 & 32 & U \\
\hline 29 & 64 & 14 & 96 & 01 & & & 5 \\
\hline & 82 & & & 02 & & & 21 \\
\hline & 82 & 14 & & 00 & 01 & 32 & $0-1$ \\
\hline 29 & 64 & 00 & 84 & 05 &, 61 & 03 & ,7 \\
\hline 14 & 82 & 14 & 12 & 00 & 0,00 & 31 & 0 \\
\hline 14 & 82 & 14 & & ,00 &, 01 & 32 & 0 \\
\hline & 82 & 14 & & 00 & 0,02 & & 07 \\
\hline 14 & 82 & 14 & 12 &, 05 & 0,67 & 54 &, 4 \\
\hline 14 & 82 & 14 & 12 & ,00 & 0,01 & 32 & 0 \\
\hline 14 & 82 & 14 & & 23 & 2,9 & & 5 \\
\hline & 82 & 2 & & & $2,3<$ & 23 & the \\
\hline 29 & 64 & 43 & & 02 & ,2 & & $x^{2}$ \\
\hline 14 & 2 & 14 & & & 0,03 & & 0 \\
\hline 14 & L & & & & & & 1 \\
\hline 14 & 82 & 29 & & Do & בנט, & & 1 \\
\hline 14 & 82 & 86 & 72 & 02 & 0,21 & 58 & 4 \\
\hline 29 & 64 & 00 & 68 & 14 & 1,80 & ,70 & 7 \\
\hline 14 & & & & & 0,01 & 32 & 0 \\
\hline 4 & 82 & 14 & & 0 & 0,01 & 32 & 1,0 \\
\hline 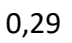 & 64 & 57 & 48 & &, 18 & 77 &, 3 \\
\hline 14 & 82 & 14 & & 01 &, 08 & 34 & 1 \\
\hline 14 & 82 & 1 & & & 05 & & 0 \\
\hline 29 & & & & & 0 & & , \\
\hline 43 & 4 & & & & 2,7 & & 22 \\
\hline 43 & 46 & 3,57 & 8 & & 4,42 & 09 & 9 \\
\hline 14 & 2 & & & & 0 & & 0 \\
\hline 14 & 2 & & & & 0 & & 1,0 \\
\hline 14 & 82 & 14 & & & 0 & & 0 \\
\hline & 82 & 43 & & & 2 & 40 & 19 \\
\hline & 2 & 10 & & & רחת & 32 &, 0 \\
\hline 14 & 2 & & & & مמת & & , \\
\hline 29 & & & & & 0 & & , \\
\hline 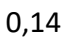 & 2 & 7 & & & 0 & & 3 \\
\hline 9 & & & & & 21 & & - \\
\hline & & & & & & & 1 \\
\hline 86 & & & & & & & ), \\
\hline 14 & 2 & 1 & & & 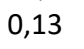 & 6 & 1 \\
\hline & 2 & & & & & & 11 \\
\hline & & & & & & & 9 \\
\hline 14 & & & & & & & ,3 \\
\hline 14 & 82 & & & & $\Omega_{1}$ & & 0 \\
\hline 57 & 8 & & & & 0 & 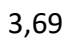 & 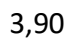 \\
\hline 14 & & & & & & & 19 \\
\hline 14 & 82 & & & & & & 1,2 \\
\hline 14 & 07 & & & & & & 1 \\
\hline 14 & 0,8 & & & & & & 0 \\
\hline 57 & & & & & 30,60 & ,71 & 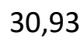 \\
\hline 29 & & & & & & & , \\
\hline & & & & & & & 3 \\
\hline 14 & & & & & & & 0 \\
\hline & & & & & $U_{1}$ & & 0 \\
\hline 43 & & & & 02 & & 34 & ,7 \\
\hline & & & & & & & 2 \\
\hline 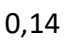 & & & & & & &, 0 \\
\hline 14 & 82 & & & 01 & ,09 & & 1 \\
\hline & & & & b1 & 08 & 34 &, 1 \\
\hline 14 & ,82 & & & 0,00 & 0,01 & 0,32 & \\
\hline
\end{tabular}

Dentre as famílias com maior densidade de indivíduos, a Lamiaceae foi a que obteve valor superior com 31,26\%, seguida da Meliaceae 15,81\%, Lauraceae 11,74\% e Fabaceae 10,30\%. As demais 20 famílias compreenderam $30,89 \%$ dos indivíduos restantes. Nenhuma espécie foi comum a todos os SAF. As espécies 
mais frequentes nos sistemas agroflorestais foram embaúba (Cecropia engleriana Snethl.) e bandarra (Schizolobium parahyba var. amazonicum (Huber ex Ducke) Barneby), estando presentes em $86 \%$ dos SAF. Seguidas por Artocarpus heterophyllus Lam., Ceiba pentandra (L.) Gaertn., Swietenia macrophylla King., Tabebuia sp., e Tectona grandis L. f., frequentes em $57 \%$ dos agroecossistemas.

O valor de importância (VI maior ou igual a 3) das espécies presentes nos SAF, mostrou um estoque de indivíduos com potencial madeireiro. Sendo elas: Tectona grandis L. f., Schizolobium parahyba var. amazonicum (Huber ex Ducke) Barneby, Swietenia macrophylla King, Mezilaurus itauba (Meisn.) Taub. ex Mez., e Tabebuia sp. Essas espécies juntas corresponderam a 62,58\% do valor de cobertura das espécies nos SAF. Quanto a estrutura vertical, os SAF apresentaram-se multiestratificados, com presença de três estratos para todos os agroecossistemas (Tabela 5). O valor fitossociológico mostrou que em $43 \%$ dos SAF os indivíduos estão melhor representados no estrato dois (E2). O SAF B, apresentou os maiores valores médios de altura por estrato. Ressalta-se que nesse agroecossistema, a espécie predominante é a bandarra (Schizolobium parahyba var. amazonicum).

Tabela 5: Distribuição dos indivíduos por média de altura, valor fitossociológico e número de indivíduos por estrato para os sete sistemas agroflorestais do município de Cacoal, Rondônia.

\begin{tabular}{|c|c|c|c|c|c|c|c|c|c|c|}
\hline \multirow[b]{2}{*}{ SAF } & \multicolumn{3}{|c|}{ Média de altura (m) } & \multicolumn{3}{|c|}{ Valor fitossociológico } & \multicolumn{3}{|c|}{ Indivíduos } & \multirow[b]{2}{*}{ Totais } \\
\hline & E1 & E2 & E3 & E1 & E2 & E3 & E1 & E2 & E3 & \\
\hline A & 7,31 & 10,42 & 13,09 & 37,64 & 22,81 & 39,54 & 99 & 60 & 104 & 263 \\
\hline B & 10,25 & 28,60 & 33,75 & 30,77 & 38,46 & 30,77 & 4 & 5 & 4 & 13 \\
\hline C & 6,15 & 8,85 & 14,16 & 39,16 & 29,37 & 31,47 & 56 & 42 & 45 & 143 \\
\hline D & 4,09 & 6,86 & 20,03 & 32,08 & 33,96 & 33,96 & 17 & 18 & 18 & 53 \\
\hline $\mathbf{E}$ & 4,41 & 7,20 & 11,65 & 28,57 & 39,10 & 32,33 & 38 & 52 & 43 & 133 \\
\hline $\mathbf{F}$ & 5,97 & 11,58 & 18,22 & 35,23 & 34,09 & 30,68 & 31 & 30 & 27 & 88 \\
\hline G & 5,11 & 8,33 & 13,50 & 28,87 & 44,37 & 26,76 & 41 & 63 & 38 & 142 \\
\hline
\end{tabular}

Onde: $\mathrm{E} 1$ = estrato inferior; E2 = estrato médio e E3 = estrato superior.

\section{DISCUSSÃO}

A relação entre a composição florística dos SAF com cafeeiro e os agricultores familiares é função de vários fatores como, por exemplo, conhecimento que os agricultores possuem sobre os potenciais usos, características culturais das famílias, espécies regenerantes e banco de sementes no solo. Assim, dissimilaridade entre os SAF pode estar associada, principalmente aos diferentes históricos de implantação, tipo e a intensidade do manejo aplicado nos agroecossistemas ao longo dos anos, mesmo que dentro da mesma propriedade. Deste modo, a diversidade dos SAF pode ser menor do que a encontrada em outros SAF (BOLFE et al., 2011), pois os agricultores priorizam o cafeeiro como cultura de interesse, manejando os demais componentes com podas e desbastes para favorecer a cultura principal, considerando o conjunto de características edafoclimáticas e socioambientais locais. Assim, os SAF fornecem produtos similares às florestas, que atendem as necessidades humanas, colaborando para a soberania alimentar dos agricultores familiares (MURNIATI et al., 2001).

Das espécies encontradas nos sete sistemas agroflorestais, 70\% ocorreram apenas uma vez. Isso, mostra a importância dos SAF para a conservação da natureza e agrobiodiversidade, como também analisado por Wojkowski (2008). No contexto Amazônico, muitas das espécies ocorrentes nos agroecossistemas 
amostrados também compõe os outros sistemas agroflorestais (BOLFE et al., 2011; MASCARENHAS et al., 2017). Atualmente, a literatura indica que a conservação de espécies vegetais por comunidades rurais está relacionada diretamente as características do grupo social (PARDO-DE-SANTAYANA et al., 2015). Deste modo, os sistemas agroflorestais na Amazônia Ocidental podem apresentar um alto potencial de conservação da natureza, pois como demonstrado nos resultados deste trabalho, por em conjunto apresentar uma quantidade significativa de espécies cultivadas, favorecendo a formação de mosaicos diversificados no ambiente rural e consequentemente a formação de corredores ecológicos e refúgios para diversas espécies (PERFECTO et al., 2005; BHAGWAT et al., 2008; BEENHOUWER et al., 2013).

A contribuição em riqueza da família Fabaceae, também já foi mencionada em outros levantamentos na Amazônia (BOLFE et al., 2011; LEÃO et al., 2017). Espécies pertencentes a essa família, apresentam capacidade de associar-se a bactérias fixadoras de nitrogênio e são amplamente citadas como componentes de áreas de SAF, projetos de recuperação de áreas degradadas, arborização urbana e paisagismo. No contexto socioeconômico e ambiental, as leguminosas são utilizadas para alimentação humana, produção de fitoterápicos, forragem animal, recuperação de áreas degradadas, produtos madeiráveis, óleos, resinas e lenha (SALVIANO, 1996).

Sistemas multiestratifados como estes colaboram com bens e serviços ecossistêmicos, manutenção da fauna, melhor qualidade de vida dos agricultores familiares e soberania alimentar (BEENHOUWER et al., 2013). Com vista as fortes pressões antrópicas sobre as áreas de florestas naturais, os SAF se fortalecem na região como uma alternativa de produção de baixo impacto, ao mesmo tempo em que promove a conservação dos recursos naturais e do conhecimento tradicional local dos agricultores familiares.

\section{CONCLUSÕES}

Os sistemas agroflorestais do município de Cacoal possuem em sua composição gradiente de espécies, sendo pouco semelhantes entre si. O SAF G apresentou os maiores valores para o índice de diversidade de Shannon-Wierner com 2,79; equabilidade de Pielou 0,87 e dominância de Simpson 0,92. 0 valor de importância das espécies que compõem os SAF apresentou grande estoque de indivíduos com potencial madeireiro.

\section{REFERÊNCIAS}

ALVARES, C. A.; STAPE, J. L.; SENTELHAS, P. C.; GONÇALVES, J. L. M.; SPAROVEK, G.. Köppen's climate classification map for Brazil. Meteorologische Zeitschrift, Stuttgart, v.22, n.6, p.711-728, 2013.

BEENHOUWER, M.; AERTS, R.; HONNAY, O.. A global metaanalysis of the biodiversity and ecosystem service benefits of coffee and cacao agroforestry. Agriculture, Ecosystems and Environment, Zurich, v.175, p.1-7, 2013.

BHAGWAT, S. A.; WILLIS, K. J.; BIRKS, H. J. B; WHITTAKER, R. J. Agroforestry: a refuge for tropical biodiversity?. Trends in Ecology and Evolution, Cambridge, v.23, n.5, p.261-267, May 2008. DOI: http://doi.org/10.1016/j.tree.2008.01.005
BOLFE, E. L.; BATISTELLA, M.. Análise florística e estrutural de sistemas silviagrícolas em Tomé-Açu, Pará. Pesquisa Agropecuária Brasileira, Brasília, v.46, n.10, p.1139-1147, 2011.

BROWER, J. E.; ZAR, J. H.. Field and laboratory methods for general ecology. 2 ed. Dubuque: Wm. C. Brown Publishers, 1984.

CASAGRANDE, B.. Caracterização do meio físico e avaliação do desmatamento no município de Cacoal - RO de 1986 a 2007, utilizando técnicas de

geoprocessamento. Dissertação (Mestrado em Geografia e 
Gestão do Território) - Universidade Federal de Uberlândia, Uberlândia, 2009.

HARRINTON, L.; TOW, P.. Types of rainfed farming systems around the word. Rainfed Farming Systems, Springer, Dordrecht, p.45-74, 2012. DOI:

https://doi.org/10.1007/978-1-4020-9132-2 2

HUTCHESON, K.. A test for comparing diversities based on the Shannon formula. Journal of Theoretical Biology, Cambridge, v.29, n.1, p.151-154, 1970. DOI: https://doi.org/10.1016/0022-5193(70)90124-4

LAMPRECHT, H.. Ensayo sobre la esttructura florística de la parte sur - oriental del bosque universitário 'El aimital', Estado Barinas. Revista Forestal Venezolana, Mérida, v.7, n.10/11, p.77-119, 1964

LEÃO, F. M.; DIONISIO, L. F. S.; SILVA, N. G. E.; OLIVEIRA, M. H. S.; D'ARACE, L. M. B.; NEVES, R. L. P.. Fitossociologia em sistemas agroflorestais com diferentes idades de implantação no município de Medicilândia, PA. Revista Agro@mbiente On-line, Boa Vista, v.11, n.1, p.71-81, 2017. DOI: http://dx.doi.org/10.18227/1982-8470ragro.v11i1.3402

LONGHI, S. J. A.. A estrutura de uma floresta natural de Araucaria angustifolia (Bert.) O. Ktze, no Sul do Brasil. Dissertação (Mestrado em Engenharia Florestal) Universidade Federal do Paraná, Curitiba, 1980.

MARTINS, S. V.; NETO, A. M.; RIBEIRO, T. M.. Uma abordagem sobre diversidade e técnicas de restauração ecológica. In: MARTINS, S. V.. Restauração ecológica de ecossistemas degradas. 2 ed. Viçosa: UFV, 2015. p.376.

MASCARENHAS, A. R. P.; SCCOTI, M. S. V.; MELO, R. R.; CORRÊA, F. L. de O.; SOUZA, E. F. M. de; ANDREDE, R. A.; BERGAMIN, A. C.; MÜLLER, M. W.. Atributos físicos e estoques de carbono do solo sob diferentes usos da terra em Rondônia, Amazônia Sul-Ocidental. Pesquisa Florestal Brasileira, Colombo, v.7, n.89, p.19-27, 2017. DOI: http://dx.doi.org/10.4336/2017.pfb.37.89.1295

MONTAGNINI, F. et al. Sistemas agroforestales: princípios y aplicaciones em los trópicos. 2 ed. San José: OET, 1992.
MUELLER-DOMBOIS, D.; ELLENBERG, H.. Aims and methods of vegetation ecology. New York: Willey e Sons, 1974.

MURNIATI, E.; GARRITY, D. P.; GINTINGS, A. N.. The contribution of agroflorestry systems to reducing farmers dependence on the resources of adjacent national parks: a case study from Sumatra, Indonesia. Agroforestry Systems, Springer Netherlands, v.52, n.3, p 171-184, 2001. DOI: https://doi.org/10.1023/A:1012047602192

OLIVEIRA, O. A.. Geografia de Rondônia Espaços e Produção. Porto Velho: Dinâmica, 2005.

PARDO-DE-SANTAYANA, M.; MACÍA, M. J.. The benefits of traditional knowledge. Nature, London, v.518, p.487-488, 2015. DOI: https://doi.org/10.1038/518487a

PERFECTO, I.; VANDERMEER, J.; MAS, A.; PINTO, L. S. Biodiversity, yield, and shade coffee certification. Ecological Economics, Hanover, v.54, n.4, p.435-446, 2005. DOI: https://doi.org/10.1016/i.ecolecon.2004.10.009

PERKINS, J. L.. Shannon-Wever or Shannon-Wiener?. Journal Water Pollution Control Federation, New York, v.54, n.7, p.1049-1050, 1982.

SALVIANO, A. A. C.. Variabilidade de atributos de solo e de Crotalaria juncea $\mathrm{L}$. em solo degradado no município de Piracicaba - SP. São Paulo. Tese (Doutorado em Agronomia) - Universidade de São Paulo, 1996.

SOARES, C. P. B.; NETO, F. P.; SOUZA, A. L.. Dendrometria e inventário florestal. 2 ed. Viçosa: UFV, 2012.

STURGES, H. A.. The Choice of a Class Interval. Journal of the American Statistical Association, Raleigh, v.21, n.153, p.6566, 1926. DOI:

http://dx.doi.org/10.1080/01621459.1926.10502161

WOJTKOWSKI, P. A.. Agrobiodiversity. In: WOJTKOWSKI, P. A.. Agroecological Economics: Sustainability and Biodiversity, Oxford: Elsevier's Science \& Technology Rights, 2008, p.45-72.

A CBPC - Companhia Brasileira de Produção Científica (CNPJ: 11.221.422/0001-03) detém os direitos materiais desta publicação. Os direitos referem-se à publicação do trabalho em qualquer parte do mundo, incluindo os direitos às renovações, expansões e disseminações da contribuição, bem como outros direitos subsidiários. Todos os trabalhos publicados eletronicamente poderão posteriormente ser publicados em coletâneas impressas sob coordenação da Sustenere Publishing, da Companhia Brasileira de Produção Científica e seus parceiros autorizados. Os (as) autores (as) preservam os direitos autorais, mas não têm permissão para a publicação da contribuição em outro meio, impresso ou digital, em português ou em tradução. 